Cahiers $d u$ MONDE RUSSE

\section{Cahiers du monde russe}

Russie - Empire russe - Union soviétique et États indépendants

$50 / 4 \mid 2009$

Varia

\title{
Niels Erik Rosenfeldt, The "Special” World
}

\section{Larissa Zakharova}

\section{OpenEdition \\ Journals}

Édition électronique

URL : https://journals.openedition.org/monderusse/7180

DOI : $10.4000 /$ monderusse. 7180

ISSN : $1777-5388$

Éditeur

Éditions de l'EHESS

Édition imprimée

Date de publication : 15 décembre 2009

Pagination : 831-836

ISBN : 978-2-7132-2261-0

ISSN : $1252-6576$

\section{Référence électronique}

Larissa Zakharova, "Niels Erik Rosenfeldt, The "Special" World », Cahiers du monde russe [En ligne], 50/4 | 2009, mis en ligne le 12 janvier 2011, consulté le 03 septembre 2022. URL : http://

journals.openedition.org/monderusse/7180; DOI : https://doi.org/10.4000/monderusse.7180

Ce document a été généré automatiquement le 3 septembre 2022.

Tous droits réservés 


\title{
Niels Erik Rosenfeldt, The "Special" World
}

\author{
Larissa Zakharova
}

\section{RÉFÉRENCE}

Niels Erik ROSENFELDT, The "Special" World. Stalin's Power Apparatus and the Soviet System's Secret Structures of Communication. Copenhague : Museum Tusculanum Press, University of Copenhagen, 2009, vol. $1: 633$ p., vol. $2: 520$ p.

Ces 1150 pages d'un historien danois sont consacrées au fonctionnement du sommet de la pyramide de l'appareil du pouvoir stalinien. L'ouvrage traite du « Secrétariat secret de Stalin », composé selon l'auteur de trois institutions : le Département secret du Comité central, le Secteur spécial du CC et le Secrétariat personnel de Stalin. Il s'agit de décrypter l'univers du secret en tant que principe du gouvernement stalinien : quels sont la structure, le fonctionnement et l'importance de cet ensemble de départements ultrasecrets qui permirent à Stalin et à son entourage de gouverner et de contrôler aussi bien la société soviétique que le mouvement communiste mondial ?

2 Ce livre est la synthèse d'une recherche dont les premières ébauches ont été publiées en 1978. L'auteur soumet ses anciennes hypothèses à l'épreuve des archives accessibles depuis le début des années 1990. Rangé du côté des historiens de l'école «totalitariste » - qu'il appelle «traditionaliste»-, il s'efforce de répondre aux critiques des " révisionnistes ». Il promeut donc une histoire politique et institutionnelle qui permet, selon lui, de reconstituer le mécanisme de prise de décision qui régit, à l'époque stalinienne, « les conditions de vie et de travail de toute la population soviétique ».

Le livre comporte huit chapitres. Le chapitre introductif présente l'historiographie du problème, les enjeux de l'ouvrage, les sources exploitées. Le deuxième détaille les principes et les procédures de transmission des informations secrètes. Les trois suivants démêlent l'écheveau des institutions au sein du Secrétariat secret, et ce dans une perspective chronologique, des années 1920 jusqu'aux années 1980. L'auteur s'arrête 
parfois sur des hypothèses impossibles à vérifier, faute d'accès aux sources. Après avoir ainsi présenté la circulation de l'information entre le centre et les régions ainsi que les sources à partir desquelles Stalin obtient des renseignements sur les pays étrangers (tel le Bureau de l'information internationale), le sixième chapitre analyse le processus de prise de décision, en essayant de comprendre par quels moyens Stalin réussit à imposer sa volonté à ses collaborateurs. La septième section porte sur les départements spéciaux et autres structures secrètes au sein de la Sécurité d'État, leur évolution structurelle et leurs domaines d'intervention. Le dernier chapitre enfin traite des structures secrètes de communication au sein du Komintern et de leurs rapports avec le secrétariat de Stalin.

4 La base documentaire du livre repose pour l'essentiel sur des matériaux du RGASPI. L'ouvrage s'appuie aussi sur d'autres sources: matériaux des interrogatoires de deux espions soviétiques employés au NKVD (archives australiennes), récits d'émigrés soviétiques, mémoires, ou encore archives de Smolensk. Les documents du département secret de la Direction des affaires du Conseil des commissaires du Peuple (GARF, f. 5446, op. 17) auraient aussi pu être utilisés, car ils concernent la tenue d'écritures secrètes et le respect du régime du secret dans les communications gouvernementales. Cela aurait été d'autant plus opportun qu'au chapitre VI, l'auteur souligne la montée en puissance des structures d'État dans la prise de décision à la veille de la guerre puis au cours de la décennie suivante.

Les questions concernant le pouvoir, le contrôle et la transmission de l'information permettent de revenir aux débats sur la nature du régime stalinien. Ainsi, l'ouvrage interroge les prémisses institutionnelles à l'exercice du pouvoir stalinien. Il décrit une forme de construction du pouvoir qui consiste à développer le système du secret. $\mathrm{Ce}$ dernier permet de contrôler le processus tout entier de communication et de diffusion de l'information à l'intérieur de l'appareil d'État. L'information est une source de pouvoir : la domination s'exerce à travers le savoir (« domination through knowledge » et « needto-know principle»). Le but de Stalin est donc de concentrer l'information entre ses mains et de doser l'accès à l'information suivant la hiérarchie institutionnelle. Le dictateur veille également à maintenir et renforcer son autorité sur la vérification de l'application des décisions. Cette manie de la vérification contribue à la formation d'un environnement où tout le monde se dénonce mutuellement, ce qui aide Stalin « dans sa lutte contre ses ennemis réels et imaginés » (vol. 1, p. 632). Au final, le livre dépeint un Stalin tout-puissant qui tient tous les fils de la communication.

6 Le culte du secret des bolcheviks prend ses racines dans les pratiques de travail dans la clandestinité de la période prérévolutionnaire : le parti centralisé fonctionne alors dans la conspiration. Ce souci du secret se renforce pendant la guerre civile dans une Russie bolchevique isolée qui doit affronter l'hostilité du monde capitaliste. La philosophie politique des bolcheviks, fondée sur la présence d'ennemis extérieurs et intérieurs, se nourrit de l'hystérie des années 1930 où les figures de l'espion et du traître sont omniprésentes. Cette obsession de la conspiration fait que, dans les années 1920, les instances dirigeantes se voient interdire de se référer au contenu des résolutions classées secrètes. De la même façon, se met en place un système d'envoi des extraits de procèsverbaux des réunions du Politbjuro et d'autres documents secrets aux responsables locaux, avec obligation de retour sous huitaine, conformément au degré de confidentialité, toute copie devant être détruite. Les originaux eux-mêmes, parfois brûlés, rendent difficile aujourd'hui le travail de l'historien. Ainsi, à l'intérieur de la sphère confidentielle, s'établit une distinction entre documents secrets « ordinaires » et 
matériaux ultrasecrets (de la série « $K »)$, conservés séparément par un personnel « de confiance ».

7 Le Bureau secret d'enregistrement et l'Expédition secrète procèdent à l'envoi des matériaux aux différentes institutions à l'aide de coursiers. Les informations les plus secrètes, codées dans un bureau spécial du Comité central, sont adressées non seulement aux instances du pouvoir à tous les niveaux, mais aussi aux partis communistes étrangers. De Soči, Stalin envoie des télégrammes codés à ses collaborateurs les plus proches au Kremlin. Ce bureau d'encodage, qui travaille de concert avec l'OGPU, s'occupe de l'élaboration, de l'enregistrement et de la transmission des codes aux instances du pouvoir.

8 C'est le Département spécial au sein de l'OGPU/NKVD/MGB, et les services qui lui succèderont, qui sont chargés des communications chiffrées. Leurs employés décodent les messages interceptés des pays étrangers et les transmettent à Stalin et au Politbjuro. Une communication étroite existe entre le Département et le Secrétariat secret de Stalin au sujet des procédures de mise en sûreté et de préservation du secret au parti et dans les organisations d'État. À l'époque de la Grande Terreur, les canaux de communication entre les services du NKVD et le Secrétariat secret de Stalin sont submergés de listes de personnes arrêtées attendant leur sentence et de rapports sur les répressions. Les directives concernant les répressions, hautement confidentielles, doivent être envoyées codées aux organes locaux.

9 En 1949, le Département spécial du NKVD se retrouve sous la tutelle directe de l'appareil central du parti, jusqu'à la création en 1954 du KGB qui abrite de nouveau le service d'encodage. D'autres départements secrets du NKVD s'occupent du " contrôle politique » (censure $\mathrm{du}$ courrier, écoutes téléphoniques), du repérage des transmissions radiophoniques illégales, de la production de faux papiers d'identité, des expériences de poisons sur les êtres humains, de l'organisation des assassinats à l'étranger ou encore de pourvoir à la sûreté personnelle des dirigeants soviétiques. On peut supposer que si l'auteur avait pu utiliser les documents de l'OGPU/NKVD/MGB, nous aurions vu non seulement l'évolution institutionnelle de ces départements, mais aussi leurs employés en action.

10 Les règles de base de la conspiration au sein du Secrétariat secret de Stalin sont également valables pour la communication à l'étranger au sein du Komintern. Les commissariats aux Affaires étrangères et au Commerce extérieur servent de courroie de transmission pour les messages du Komintern. Les agents résidant à l'étranger se présentent en tant que commerciaux, membres de compagnies d'import-export. Ils organisent des rencontres clandestines dans des appartements secrets. L'appareil de communication secrète du Komintern (le Département de communication internationale puis le Service de communication) est chargé de transmettre instructions, informations et financements aux partis frères à l'étranger. Il a par ailleurs un compte secret dans la Banque d'État ouvert sous un faux nom. Ce service s'occupe aussi de la coordination des activités de Komintern liées à l'espionnage. Toutes les décisions liées aux activités du Komintern sont envoyées à Stalin pour approbation. Après la dissolution du Komintern en 1943, un énigmatique Institut $n^{\circ} 100$ sera chargé entre autres de la soviétisation de l'Europe orientale.

11 Le Secrétariat secret de Stalin est ainsi le centre de commandement du réseau entier de communication confidentielle, dont le nombre d'employés ne cesse d'augmenter : de douze au début des années 1920, il atteint la centaine de personnes à la fin de la décennie. 
Or, à la fin des années 1920, le champ de confidentialité s'est étendu à des domaines qu'il ne couvrait pas jusqu'alors, telle l'agriculture. Néanmoins, les questions de politique étrangère, de défense et de sécurité d'État restent de première importance. Les mesures de préservation du secret sont renforcées: toutes les résolutions du parti et du gouvernement sont considérées comme secrètes, sauf mention contraire expresse.

Le Secteur spécial, un nouveau secrétariat du Politbjuro, est officiellement créé au sein de l'appareil secret du parti en 1934, avec à sa tête le premier assistant de Stalin, Aleksandr Poskrëbyšev. L'auteur cherche à nous convaincre que ce secteur est apparu quelques années plus tôt, lors de la vague de la campagne de mobilisation pour la guerre. Ce ne serait pas un simple changement d'appellation - le Département secret devenant le Secteur spécial, mais une véritable réorganisation avec une nouvelle redistribution des fonctions à l'intérieur de l'appareil secret : le Comité central et le Politbjuro ont désormais chacun leurs secrétariats confidentiels. C'est au Secteur spécial que reviennent l'envoi et la conservation des documents de la plus haute confidentialité : matériaux compromettants, questions de mobilisation et de sécurité. Les types de documents présentés révèlent quels sujets préoccupaient Stalin. Ces fluctuations au sommet entraînent l'apparition de secteurs spéciaux dans chaque organisation régionale du parti. Conformément au modèle du Kremlin, les chefs de ces secteurs deviennent les assistants personnels des premiers secrétaires locaux.

Les derniers changements structurels interviennent en 1952, à l'occasion du XIX Congrès du parti, avec la disparition du Politbjuro et la création du Présidium. Contrairement au Secteur spécial qui fonctionne indépendamment du secrétariat du Comité central, le Département général regroupe les informations secrètes du Comité central et du Présidium. Par la suite, jusqu'aux années 1980, le Département général continuera à fonctionner sur les principes de son prédécesseur, le Secteur spécial : toute l'information destinée au Comité central, au Politbjuro et aux autres départements passe par le Département général. Les comptes rendus sténographiques des réunions du Comité central sont « corrigés » par le Département général et le résultat final correspond assez peu à ce qui a été dit par les intervenants. Les assistants du Secrétaire général continuent à briefer celui-ci sur des questions diverses et variées, profitant de leurs liens avec les membres du Comité central, les ministères, les chefs militaires, diplomates, écrivains et artistes. Ce sont eux qui définissent l'agenda général des réunions du Politbjuro.

Aussi l'auteur évoque-t-il à plusieurs reprises le rôle des assistants sur lesquels Stalin s'appuie pour gérer les documents secrets. Ces assistants préparent des dossiers, rédigent les brouillons des discours. Reprenant les débats historiographiques, l'ouvrage s'interroge sur l'influence qu'exercent certains individus, comme Lev Mehlis ou encore Aleksandr Poskrëbyšev, sur Stalin, ou du moins sur le degré du filtrage de l'information qu'ils transmettent au Secrétaire général. Poskrëbyšev - gatekeeper - selon l'auteur (terme emprunté aux théories de la communication) apparait comme un homme aux capacités surhumaines, parcourant quotidiennement des centaines de documents pour les présenter à Stalin sous forme synthétique. Son poids est évalué comme beaucoup plus important que celui des membres du Politbjuro les plus proches de Stalin. Toute la documentation destinée à ce dernier, y compris les lettres des Soviétiques adressées au Secrétaire général, passe entre ses mains.

Notons, parmi les conseillers de Stalin, l'économiste Evgenij Varga, successivement responsable du Bureau de l'information statistique à Berlin de 1921 à 1927, puis, après la fermeture de ce dernier, directeur d'un observatoire du monde capitaliste, l'Institut de la 
politique et de l'économie mondiales à Moscou. À partir du milieu des années 1930, Stalin préfère s'appuyer sur ses informateurs à l'étranger (les "correspondants spéciaux » des journaux comme Il'ja Erenburg) pour avoir des informations autres que celles fournies par les diplomates. Certains assistants et informateurs de Stalin ont échappé à la Grande Terreur, comme Varga, Poskrëbyšev, Hodov, Čërnuha, Loginov, ou encore Elena Stasova, chef du Bureau d'information et responsable de la communication avec les partis communistes étrangers dans les années 1920-1930, qui n’a pas connu le triste sort de son collègue Karl Radek du Bureau de l'information internationale.

Tout en indiquant les limites du travail de l'historien sur le sujet, l'auteur parvient, malgré le défaut d'accès aux archives, à reconstituer les processus de prise de décision sous Stalin. Paradoxalement, le nombre de sujets secrets traités lors des réunions du Politbjuro augmente considérablement au cours des années 1920-1930, tandis que la fréquence annuelle des réunions chute. L'Orgbjuro et le Secrétariat du Comité central se réunissent d'ailleurs de moins en moins souvent. Ces tendances prouvent la centralisation du processus de décision. Le paradoxe s'explique : de décisions prises lors des réunions du Politbjuro, on passe aux décisions prises hors réunion, au nom du Politbjuro, par le quintet - Stalin, Molotov, Kaganovič, Ordžonikidze et Vorošilov. Cependant, pendant la période, des commissions spécialisées au sein du Politbjuro et du CC s'occupent de domaines spécifiques (départs à l'étranger, questions urgentes d'économie, etc.). L'auteur s'interroge donc sur la nécessité de formaliser la prise de décision dans le contexte de concentration du pouvoir au sein du quintet, qui s'élargit après la guerre pour inclure de six à neuf personnes. Il en conclut que ces commissions sont nécessaires pour passer d'une prise de décision collégiale au sein du Politbjuro à des réunions plus informelles. Celles-ci n'ont pas d'agenda fixé à l'avance et ne donnent pas lieu à des comptes rendus sténographiques.

17 À partir de la correspondance de Stalin avec ses collaborateurs, l'auteur constate que ces derniers n'ont pas beaucoup d'influence sur la décision et se plient à la volonté de Stalin, non seulement par peur, mais aussi parce que c'est lui le mieux informé sur toutes ces questions. Ainsi le Politbjuro a-t-il un rôle assez marginal dans ce domaine au cours des années 1930-1940. Le mécanisme de fonctionnement pendant le premier et le second stalinisme est résumé ainsi : un membre de gouvernement ou du Politbjuro, après un entretien avec Stalin, lui envoie un brouillon concernant la décision. Stalin, qui en discute avec son assistant, y apporte des corrections. La décision peut ensuite être transmise au Conseil des ministres ou au Politbjuro pour approbation. Mais comme Stalin a déjà tout décidé, cette procédure n'est qu'une simple formalité. Dans d'autres cas, il n'y a pas d'approbation officielle: les personnes concernées sont informées par téléphone ou reçoivent la décision annotée par Stalin.

18 Afin de mieux guider le lecteur dans les méandres du pouvoir stalinien et dans les évolutions institutionnelles, l'ouvrage offre un organigramme des instances au sein du Secrétariat secret, des services de la Sûreté d'État et du Komintern. Les biographies et de nombreuses photographies originales constituent un des atouts du livre. La bibliographie contient des travaux en anglais, russe, français, allemand et danois. Dans une si belle édition, on regrettera que les notes soient regroupées à la fin du second volume, ce qui rend la consultation laborieuse. Et si le volume de l'ouvrage est imposant, c'est en partie à cause des répétitions dues au cadre chronologique du récit des chapitres III, IV et V. Il reste que le livre sera très utile à tous ceux qui s'intéressent aux 
changements institutionnels dans la pyramide du pouvoir stalinien comme à la matérialité des pratiques de secret dans l'appareil administratif soviétique. 